\title{
SUPERLUMINAL MOTION IN THE LARGEST KNOWN QUASAR
}

\author{
P. D. Barthel ${ }^{1,2}$, R. T. Schilizzi ${ }^{3}$, G. K. Miley ${ }^{2,4}$, E.Preuss ${ }^{5}$, J. R. Hooimeyer ${ }^{2}$ \\ 1. California Institute of Technology; 2. Leiden Observatory; 3. Netherlands Foundation for Radio \\ Astronomy; 4. Space Telescope Science Institute; 5. Max-Planck-Institut für Radioastronomie
}

\begin{abstract}
We discuss VLBI observations of the bright core in the large, triple radio source 4C 34.37, associated with a quasar at $z=0.206$. We have discovered superluminal motion in this quasar, which is the largest quasar known. We comment on the implications of these findings, and subsequently examine the projected size distributions of lobe and core dominated quasars in general. The results of these investigations lead us to conclude that neither superluminal motion, nor core prominence can simply be translated into an orientation of the overall radio source.
\end{abstract}

Superluminal motion, (curved) one-sided parsec scale jets, and rapid variability in compact radio sources have found a more or less satisfactory explanation in relativistic effects, resulting from a relativistic beam observed from a suitable direction (e.g., Zensus \& Pearson, these Proceedings). Along these lines of thought, attempts have also been made to unify the properties of extended, lobe dominated, and compact, core dominated radio sources (e.g., Orr \& Browne 1982), in which the morphologies and relative occurrences of these sources are explained as purely an orientation effect. Defining the simple beaming model as having unidirectional, uniform velocity, bulk relativistic motion in a jet along the overall source axis, we will in the following comment on its applicability, to explain superluminal motion and as the basis for unifying schemes.

To test the model, the occurrence of superluminal motion in the cores of large extended triple quasars, supposedly oriented near the sky plane, is of prime importance. For example, in a randomly oriented sample, one out of four extended quasars should be within $15^{\circ}$ of the sky plane, whereas according to the simple beaming model an apparent velocity of $2 c$ is impossible in this source orientation. Motivated by an increasing number of superluminal quasars with extended morphologies Barthel et al. (1986) pointed out that the simple beaming hypothesis might be inadequate to explain the observations. In the meantime, a project to study the structure and evolution of the core morphologies in very large triple quasars (Barthel et al. 1984) has resulted in another superluminal quasar, which happens to be the largest quasar known $(560 / h \mathrm{kpc}$, $H_{0}=100 \mathrm{hm} \mathrm{s}^{-1} \mathrm{Mpc}^{-1}$ and $q_{0}=0.5$ ). Fig. 1 shows a montage of the large and small

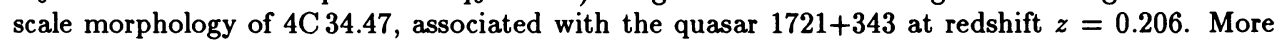
maps can be found in Barthel (1987). Combining 5 and $10.7 \mathrm{GHz}$ VLBI data we find that the northernmost core component has an inverted radio spectrum, whereas the other components have steep spectral indices. We therefore identify this northernmost component with the actual radio core, and measure $\beta_{a p p}=3.1 / h$ for the two components south of the core: $4 \mathrm{C} 34.47$ is a superluminal quasar. With $H_{0}=100$ this observation implies that the quasar lies within a $36^{\circ}$ cone with the line of sight. The chance for a randomly oriented quasar to be within this cone is $19 \%$. Unless $4 \mathrm{C} 34.47$ is a freak, we would therefore expect at least five larger quasars. We 
conclude that superluminal motion cannot simply be translated into an orientation of the overall radio source.

This 'uncomfortable' (Barthel 1987) situation led us to investigate the extent of another prediction of the simple beaming model: using similar projection arguments as above, core dominated quasars should be smaller in projected linear size than lobe dominated quasars. To this end we extracted quasars with known flux densities for their core and extended emission, and available radio maps, from the compilation of Wills \& Browne (1986). Based on their core prominence, we divided these sources in two samples: a core dominated sample having $0 \leq \log R<1$, and a lobe dominated sample having $-1 \leq \log R<0$. Median values for the luminosities of the unbeamed (lobe) emission were the same for both samples (important criterion - we don't want to compare apples and pears!). It is easy to show that the simple beaming model $\left(\mathrm{R}_{T}=0.024\right.$ and $\left.\gamma=5\right)$ predicts that our core dominated sample should have half the projected linear extent of the lobe dominated sample. The actual size distributions, shown in Fig. 2, indicate that this is definitely not the case: the two distributions are statistically undistinguishable. Apparently both lobe and core dominated quasars can display compact and extended morphologies. Similar results have been obtained by Murphy (Ph. D. thesis, as reported in Browne, 1987). We conclude that core prominence cannot simply be translated into an orientation of the overall source.

In summary, it seems hard to reconcile both superluminal motion and core prominence with a preferred orientation of the overall radio source axis. Maintaining the beaming hypothesis, we have to conclude that superluminal motion and flux boosting can be observed from a fairly wide range of directions, and a wide jet cone angle or oblique shocks may be relevant in this respect. The question as to the existence of unbeamed quasars was addressed at length in Barthel (1987). On the other hand, orientation independent mechanisms such as screens or intersecting shocks may apply, and core prominence may have an intrinsic, physical origin. Conclusive statements will have to be based on statistical studies of large, carefully matched samples of core and lobe dominated quasars.

\section{References}

Barthel, P.D., Miley, G.K., Schilizzi, R.T., Preuss, E. 1984: Astron. Ap. 148, 243.

Barthel, P.D., Pearson, T.J., Readhead, A.C.S., Canzian, B.J. 1986: Ap. J. (Letters) 310, L7.

Barthel, P.D. 1987: in Superluminal Radio Sources, ed. Zensus \& Pearson (Cambridge University Press), p. 148. Browne, I.W.A. 1987: in Superluminal Radio Sources, ed. Zensus \& Pearson (Cambridge University Press), p. 129. Orr, M.J.L., Browne, I.W.A. 1982: M. N. R. A. S. 200, 1067.

Wills, B.J., Browne, I.W.A. 1986: Ap. J. 302, 56.

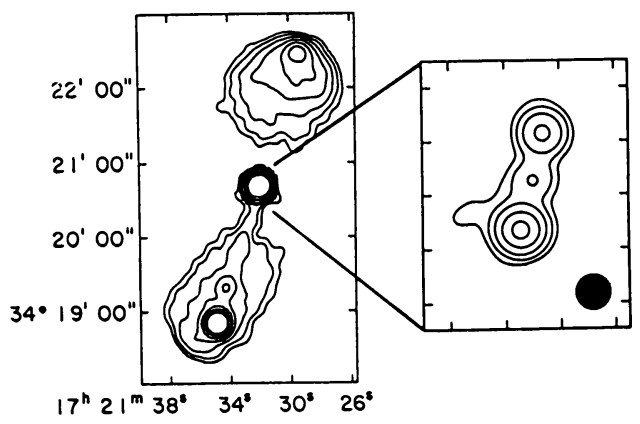

Fig. 1. Large and small scale morphology of $4 \mathrm{C} 34.47$

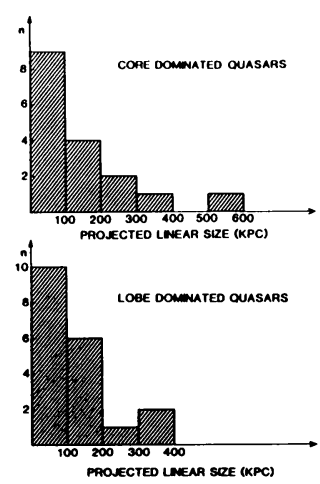

Fig. 2. Projected linear size distributions 